\title{
Lineamientos del Colegio Colombiano de Terapia Ocupacional para la atención hospitalaria aguda y subaguda de pacientes con COVID-19
}

Guidelines of the Colombian College of Occupational Therapy for acute and subacute hospital care of patients with COVID-19

Diretrizes do Colégio Colombiano de Terapia Ocupacional para atendimento hospitalar de pacientes com COVID-19 na fase aguda e subaguda

\author{
Pamela Talero Cabrejo ${ }^{1}$ \\ Francy Guacaneme García ${ }^{2}$ \\ Ruth Montufar Dulce ${ }^{3}$ \\ María Helena Rubio Grillo \\ Viviana Marcela León Perilla ${ }^{5}$ \\ Laine Yolanda Beltrán 6 \\ Sylvia Cristina Duarte Torres ${ }^{7}$
}

Recibido: 16 de mayo 2020 • Enviado para modificación: 10 de agosto 2020 • Aceptado: 15 de agosto 2020

Talero-Cabrejo, P., Guacaneme-García, F., Montufar-Dulce, R., Rubio-Grillo, M.H., León-Perilla, V., Beltrán, L. y Duarte-Torres, S. (2020). Lineamientos del Colegio Colombiano de Terapia Ocupacional para la atención hospitalaria aguda y subaguda de pacientes con COVID-19. Revista Ocupación Humana, 20 (1), 124-145. https://doi.org/10.25214/25907816.951

\footnotetext{
1 Terapeuta ocupacional. Doctora en Terapia Ocupacional. Docente, Thomas Jefferson University. Filadelfia, Estados Unidos de América. Pamela.Talero@jefferson.edu iD https://orcid.org/0000-0002$\underline{1057-9736}$

${ }^{2}$ Terapeuta ocupacional. Centro de Cuidado Clínico de Excelencia Hidrocefalia con Presión Normal, Fundación Santa Fe de Bogotá. Bogotá, Colombia. yadydd@yahoo.com iD https://orcid.org/0000-0001$\underline{7880-1914}$

${ }^{3}$ Terapeuta ocupacional. Fundación Santa Fe de Bogotá. Bogotá, Colombia. rmontufar@yahoo.com (iD) https://orcid.org/0000-0003-2660-8850

${ }^{4}$ Terapeuta ocupacional. Especialista en Desarrollo Pedagógico. Magíster en Educación. Docente, Universidad del Valle. Cali, Colombia. maria.rubio@correounivalle.edu.co iD https://orcid.org/0000-0002$\underline{3612-7885}$

${ }^{5}$ Terapeuta ocupacional. Magíster en Terapia Ocupacional. Candidata a Doctora en Terapia Ocupacional. Hospital Universitario Nacional de Colombia. Bogotá, Colombia vivianaleonperilla.to@gmail.com

(iD) https://orcid.org/0000-0003-2323-921X

${ }^{6}$ Terapeuta ocupacional. Hospital Universitario Clínica San Rafael. Bogotá, Colombia. Lybeltran1@hotmail.com iD https://orcid.org/0000-0003-2045-6067

7 Terapeuta ocupacional. Magíster en Bioética. Docente, Universidad Nacional de Colombia. Bogotá, Colombia. scduartet@unal.edu.co@iD https://orcid.org/0000-0002-0516-2696
} 


\section{RESUMEN}

El amplio espectro clínico de la enfermedad COVID-19 tiene importantes implicaciones funcionales para el cuidado hospitalario agudo y subagudo en unidades de cuidado intensivo. Ante la emergencia sanitaria desencadenada por esta enfermedad, el Colegio Colombiano de Terapia Ocupacional convocó a un grupo de profesionales expertas en el área para proveer lineamientos para terapeutas ocupacionales que participan en la rehabilitación temprana de pacientes con COVID-19 en Colombia. Se realizó una revisión de literatura sobre la clasificación y las manifestaciones clínicas de la enfermedad, la efectividad de la rehabilitación temprana adulta, pediátrica y neonatal, y el rol y la experiencia de profesionales en Terapia Ocupacional en el uso de guías de salud existentes. Un consenso de expertas determinó la aplicabilidad de los lineamientos al contexto colombiano. Terapia Ocupacional, como parte del equipo de rehabilitación temprana, juega un papel fundamental en la restauración y el mantenimiento de la independencia funcional; la adaptación del ambiente y el acondicionamiento holístico gradual de las funciones respiratorias, cardiovasculares, sensoriomotoras y cognitivas durante las actividades básicas cotidianas, y la prevención del deterioro físico, mental, espiritual y social de la persona hospitalizada y su familia. Estos lineamientos constituyen un recurso técnico y educativo relevante para la Terapia Ocupacional hospitalaria.

\section{PALABRAS CLAVE}

Terapia Ocupacional, cuidado intensivo, COVID-19, hospital, servicios de rehabilitación

\section{ABSTRACT}

The broad clinical spectrum of COVID-19 disease has critical functional implications for acute and subacute hospital care in intensive care units. Faced with the health emergency triggered by this disease, the Colombian College of Occupational Therapy convened a group of professional experts in the area to provide guidelines for occupational therapists involved in the early rehabilitation of patients with COVID-19 in Colombia. A literature review was conducted on the classification and clinical manifestations of the disease, the effectiveness of early adult, pediatric and neonatal rehabilitation, and the occupational therapist's role and experience in the use of existing health guidelines. A consensus of experts determined the applicability of the guidelines to the Colombian context. Occupational Therapy, as part of the early rehabilitation team, plays a fundamental role in restoring and maintaining functional independence, the adaptation of the environment and the gradual holistic conditioning of respiratory, cardiovascular, sensorimotor and cognitive functions during basic daily activities, and the prevention of physical, mental, spiritual and social deterioration of the hospitalized person and their family. These guidelines constitute a relevant technical and educational resource for hospital Occupational Therapy.

\section{KEY WORDS}

Occupational Therapy, intensive care, COVID-19, hospital, rehabilitation services

\section{RESUMO}

O amplo espectro clínico da doença COVID-19 tem implicações funcionais relevantes para os cuidados hospitalares agudos e subagudos em unidades de terapia intensiva. Diante 
da emergência sanitária desencadeada por esta doença, o Colégio Colombiano de Terapia Ocupacional reuniu um grupo de profissionais, especializados na área, para fornecer orientações aos terapeutas ocupacionais, envolvidos na reabilitação precoce de pacientes com COVID-19, na Colômbia. Foi realizada uma revisão da literatura sobre a classificação e as manifestações clínicas da doença, a eficácia da reabilitação adulta precoce, pediátrica e neonatal, bem como o papel e experiência do terapeuta ocupacional no uso das diretrizes de saúde existentes. Um consenso de especialistas determinou a aplicabilidade das diretrizes ao contexto colombiano. A Terapia Ocupacional, como parte da equipe de reabilitação precoce, desempenha um papel fundamental na restauração e manutenção da independência funcional; a adaptação do meio ambiente e o condicionamento holístico gradual das funções respiratórias, cardiovasculares, sensório-motoras e cognitivas, durante as atividades diárias básicas; e a prevenção da deterioração física, mental, espiritual e social da pessoa hospitalizada e sua família. Essas orientações constituem um recurso técnico e educacional relevante para a Terapia Ocupacional hospitalar.

\section{PALAVRAS-CHAVE}

Terapia Ocupacional, cuidado intensivo, COVID-19, hospital, serviços de reabilitação

\section{Introducción}

La atención hospitalaria aguda se refiere a los cuidados prestados a personas con síntomas que requieren de una intervención clínica o servicios especializados no disponibles en otro contexto de la atención en salud, desde la admisión al hospital hasta la relativa estabilidad de los signos vitales y la condición de salud. En esta fase puede requerirse atención en la unidad de cuidados intensivos (en adelante, UCI) (Coelho Filho, 2000). Por otro lado, la atención hospitalaria subaguda es el estadio que sigue a la fase aguda, en la cual la o el paciente presenta síntomas clínicos más discretos, con un pronóstico más estable, pero aún requiere de equipos e instrumentos especializados, generalmente en una $\mathrm{UCl}$ o en una unidad de cuidados intensivos intermedios (en adelante, UCl-intermedio) (Sabartés et al., 2009).

Así pues, las UCl y las UCl-intermedio son entornos intrahospitalarios espe- cializados en la atención de pacientes con condiciones clínicas críticas, agudas y subagudas, derivadas de un evento traumático, la progresión de una enfermedad o la aparición de una nueva afección. Estas unidades se caracterizan por una mayor intensidad en el cuidado clínico; un monitoreo constante de las y los pacientes (incluyendo monitores internos y externos del corazón y el cerebro); más horas de consulta de diferentes especialidades, y un mayor número de intervenciones tales como el uso de ventiladores mecánicos, soluciones intravenosas potentes, medicamentos y otras medidas terapéuticas. Todo ello, con el objetivo de recuperar o estabilizar la condición de salud de la persona (Popovich, 2011).

Gracias a los avances tecnológicos y farmacológicos, en los últimos años se ha incrementado la supervivencia de pacientes con enfermedades de diferen- 
te índole que reciben atención en $\mathrm{UCl}$ y UCl-intermedio. Sin embargo, después de una estancia en tales unidades, las personas experimentan el deterioro de su calidad de vida, con nuevos impedimentos físicos, cognitivos y de salud mental (Schweickert et al., 2009; Algeo y Aitken, 2019; Hollinger et al., 2019; Ferrante et al., 2019; Rengel et al., 2019), lo que también afecta a sus familias e incide en el sistema de salud. Así, las necesidades de atención después de la estancia en $\mathrm{UCl}$ pueden extenderse hasta por cinco años y generar altos costos, de manera que el abordaje temprano por parte del equipo de rehabilitación resulta fundamental (Rengel, et al., 2019).

El amplio espectro clínico de la enfermedad COVID-19 tiene implicaciones funcionales de gran importancia para la atención hospitalaria aguda y subaguda por parte de los equipos de rehabilitación temprana en UCl. Hasta la fecha, no existen en el país lineamientos consensuados para terapeutas ocupacionales que se involucren en la rehabilitación temprana de pacientes con COVID-19 en Colombia. Por lo tanto, el proposito de este artículo es proporcionar evidencia sobre el rol de la Terapia Ocupacional en el cuidado agudo y subagudo, centrándose en la rehabilitación temprana en $\mathrm{UCl}$ y UCl-intermedio de pacientes confirmados o sospechosos de COVID-19.

\section{Metodología}

Se realizó una revisión de la literatura internacional entre enero y mayo de 2020, a través de las bases de datos CINAHL, Scopus, Google Scholar, PubMed y OT-
Seeker, por parte de las autoras. Los descriptores principales seleccionados para la búsqueda fueron: COVID-19, virus SARS-Cov-2, hospitalización, unidad de cuidado intensivo, rehabilitación, Terapia Ocupacional, actividades de la vida diaria y acondicionamiento, de acuerdo con las características, la clasificación, y las manifestaciones clínicas de la enfermedad COVID-19; la efectividad de la rehabilitación temprana en las $\mathrm{UCI}$ y UCl-intermedio adulta, pediátrica y neonatal, y el rol y la experiencia de terapeutas ocupacionales en las guías de salud existentes. Se realizó la propuesta de lineamientos para terapeutas ocupacionales en la atención hospitalaria aguda y subaguda de pacientes con COVID-19, siguiendo un proceso iterativo de revisiones colaborativas en Google Drive entre el grupo de terapeutas ocupacionales expertas convocadas por el Colegio Colombiano de Terapia Ocupacional. Se realizaron tres reuniones virtuales de discusión y socialización con otros equipos de expertos trabajando en los lineamientos para la rehabilitación intrahospitalaria y ambulatoria, con una duración aproximada de dos horas cada una, utilizando las plataformas Zoom y GoToMeeting. A partir de las discusiones en las reuniones, se realizaron ajustes a los lineamientos para su aplicabilidad en las instituciones de salud en el contexto colombiano.

\section{Rehabilitación temprana en UCI y UCl-intermedio de población adulta y adulta mayor}

El objetivo de la rehabilitación temprana es promover el aumento de habi- 
lidades y la reanudación de las actividades básicas de la vida diaria $-A B V D^{8}$ en personas con lesiones o enfermedades agudas y críticas, teniendo en cuenta sus impedimentos, las limitaciones y demandas de la actividad ${ }^{9}$, así como las restricciones en la participación que experimentan. Hasta ahora, la rehabilitación temprana multidisciplinar, que incluye Terapia Ocupacional, ha demostrado su efectividad en el restablecimiento de las funciones sensoriomotoras, cognitivas, espirituales y socioemocionales, mejorando la independencia funcional a través de la movilización temprana, la estimulación sensorial, el cuidado de la piel, la prevención y el tratamiento de edemas y contracturas (Schweickert et al., 2009; Popovich, 2011; Alvárez et al., 2017; Costigan et al., 2019; Moreno-Chaparro et al., 2019); en la disminución de la probabilidad de desarrollar síndrome de debilidad adquirida en $\mathrm{UCI}(\mathrm{DAUCl})^{10}$ (Anekwe et al., 2019); en la reducción del tiempo de ventilación mecánica requerido (Schweickert et al., 2009; Weinreich et al., 2017; Costigan et al., 2019); en la prevención y el tratamiento del delirio, mejorando el desempeño funcional cognitivo (Álvarez et al., 2017; Cuevas-Lara et al., 2019; Pozzi et al., 2020); en la prevención de caídas (Pozzi et al., 2020); en el aumento de la participación en actividades básicas de la vida diaria
(Weinreich et al., 2017; Costigan et al., 2019), y en la reducción del tiempo de estancia hospitalaria (Needham et al., 2012; Corcoran et al., 2017).

\section{Rehabilitación temprana en $\mathrm{UCI}$ pediátrica y $\mathrm{UCI}$ - intermedio pediátrica}

El aumento de la supervivencia de pacientes en cuidados críticos ha permitido un cambio de enfoque, de prevención de la mortalidad a la funcionalidad a largo plazo; esto aplica para personas adultas, niñas y niños. Así pues, al tiempo que disminuye la mortalidad, aumenta la proporción de niñas y niños que son dados de alta con una discapacidad sustancial. La disfunción motora es la principal morbilidad (Hanna et al., 2020), seguida por el delirio infantil hospitalario; respecto a este último, en niñas y niños hospitalizados en UCl pediátrica la prevalencia general es de más del $40 \%$ y alcanza hasta el $60 \%$ en menores de 5 años, incluidos lactantes (Smith y Williams, 2020), afectando especialmente a quienes ya presentaban retardos en el desarrollo (Kaur et al., 2020).

Por otro lado, los niños y niñas que sobreviven a una enfermedad crítica

\footnotetext{
${ }^{8} \mathrm{Se}$ entiende como actividades basicas de la vida diaria, aquellas acciones dirigidas al cuidado del propio cuerpo, también conocidas como actividades personales de la vida diaria, actividades básicas cotidianas o de autocuidado (baño, aseo, vestido, alimentación, continencia, etc.) (Legg et al., 2006; Lesende et al., 2012; AOTA, 2014).

${ }^{9}$ Se entiende actividad como una categoría de acción o forma de obrar construida y compartida culturalmente (Pierce, 2001). La actividad es vista como un elemento que promueve el encuentro y el diálogo entre el sujeto, su grupo social, su época histórica y su tradición cultural (Lima et al., 2013).

${ }^{10} \mathrm{El}$ síndrome de debilidad adquirida en $\mathrm{UCl}(\mathrm{DAUCl})$ se caracteriza por una disminución de la fuerza muscular que por lo general se asocia a atrofia; de comienzo agudo, difuso, simétrico y generalizado; se desarrolla luego del comienzo de una enfermedad crítica, sin otra causa identificable. Suele manifestarse de manera bilateral en las extremidades con hiporreflexia o arreflexia y preservación de los nervios craneales (Anekwe et al., 2019).
} 
corren el riesgo de desarrollar afecciones significativas y duraderas, las cuales pueden incluir debilidad neuromuscular, deficiencias cognitivas y trastornos de salud mental (Wieczorek et al., 2016). Estas morbilidades, conocidas en su conjunto como síndrome poscuidados intensivos pediátricos, se manifiestan en forma de disfunciones físicas, emocionales y neurocognitivas que pueden persistir por mucho tiempo después de que es dado de alta, afectando también a sus cuidadores (Herrup et al., 2017).

El efecto de la enfermedad crítica en la función general del niño o niña, en la calidad de vida y en la reintegración al hogar y a la escuela, así como el potencial de recuperación justifican de manera contundente la rehabilitación temprana (Hopkins et al., 2015). Aunque la literatura relacionada con las prácticas de rehabilitación en UCI pediátrica apenas está emergiendo, la información disponible indica que las actividades de movilización temprana en niños y niñas en estado crítico han demostrado ser seguras y factibles, y que pueden traer beneficios a corto y largo plazo (Wieczorek et al., 2016; Betters et al., 2017; Cuello-García et al., 2018; Fink et al., 2019; Hanna et al., 2020).

Por lo tanto, las y los terapeutas ocupacionales en la UCI pediátrica, al considerar todas las áreas del desempeño, principalmente el juego, la influencia del contexto hospitalario y las co-ocupaciones $^{11}$ de infantes y cuidadores, están en capacidad de diseñar intervenciones centradas en la familia. Así, haciendo uso de actividades que se ajustan al desarrollo físico, cognitivo, emocional y social de las y los pequeños, educan y alientan a las familias a aprender y aplicar intervenciones de rehabilitación apropiadas, especialmente cuando no se cuenta con recursos o condiciones para acceder a servicios rehabilitación después del egreso de la unidad (Liang et al., 2017; Berrios et al., 2019).

\section{Rehabilitación temprana en UCI neonatal y UCI-intermedio neonatal}

Aunque los avances recientes en el desarrollo tecnológico y en las prácticas terapéuticas y quirúrgicas en las unidades de cuidados intensivos neonatales han aumentado las tasas de supervivencia de recién nacidos que requieren este tipo de atención, la evidencia muestra una gran cantidad de alteraciones del desarrollo entre aquellos que superan la estancia en dichos servicios (Martell et al., 2007; Borges Nery et al., 2019). Las y los recién nacidos prematuros tienen un mayor riesgo de presentar problemas en el desarrollo o una enfermedad mental a largo plazo, como trastorno por déficit de atención e hiperactividad, dificultades del aprendizaje, retrasos motores, problemas de percepción visual y viso-motores, déficit del funcionamiento ejecutivo, parálisis cerebral y problemas de visión y audición (Ross et al., 2017; Yates et al., 2020). Además, el $74 \%$ de bebés con peso extremadamente bajo al nacer experimentan alteraciones en los resultados funcionales a los

\footnotetext{
${ }^{11}$ La co-ocupación se refiere a la ocupación realizada entre dos o más personas al mismo tiempo y en la cual se comparten aspectos físicos, emocionales, así como una intencionalidad y un significado (Pickens et al., 2009).
} 
cinco años (Ross et al., 2017). De esta manera, se estima que las y los recién nacidos con estancias prolongadas tienen mayor prevalencia de enfermedades crónicas, deterioro cognitivo, alteraciones en el neurodesarrollo y alta mortalidad (Benavidez, 2006).

Por otro lado, las madres y los padres de neonatos que ingresan a la $\mathrm{UCI}$ neonatal experimentan niveles elevados de estrés y tienen un mayor riesgo de desarrollar problemas de salud mental, incluso después de que sus hijas o hijos sean dados de alta (Carter et al., 2005; Treyvaud et al., 2014; Marrugo-Arnedo et al., 2019). Adicionalmente, la indispensable priorización de las necesidades clínicas de las y los recién nacidos prematuros en la $\mathrm{UCl}$ neonatal resulta en la interrupción del rol de madre y cuidadora (Smith et al., 2019).

Así pues, la intervención de Terapia Ocupacional en la UCI neonatal se centra en reducir las condiciones que resultan estresantes para la o el recién nacido, debido a la inmadurez fisiológica de sus diversos sistemas corporales, incluido el sistema nervioso. La intervención terapéutica y la orientación a los padres permiten identificar condiciones ambientales que provocan desequilibrio o estrés para la o el bebé; de esta manera, la Terapia Ocupacional proporciona herramientas importantes y necesarias para responder a sus necesidades desde un abordaje integral, centrado en la familia y reconociendo la interacción entre el desarrollo y el ambiente (Hernández et al., 2016). Así mismo, facilita la maduración del sistema neurológico y la adecuada integración sensorial del bebé, teniendo en cuenta que, en el caso de recién nacidos pretérmino, sus sistemas sensoriales inmaduros no están prepa- rados para responder a los estímulos del ambiente. Mediante su intervención, las y los terapeutas ocupacionales fomentan la estabilidad fisiológica, la serenidad, la vitalidad y la capacidad del neonato de interactuar con su entorno físico y humano (Borges Nery et al., 2019; Rubio GriIlo, 2019; Smith et al. 2019).

\section{Rehabilitación temprana y desafíos en pacientes con COVID-19}

La enfermedad infecciosa COVID-19, causada por un nuevo coronavirus (SARS-CoV-2), es sin duda un desafío para el gremio de la salud. El espectro clínico de la COVID-19 ha demostrado variar desde formas asintomáticas o paucisintomáticas, condiciones clínicas caracterizadas por insuficiencia respiratoria que cursa en ocasiones con disfunción cardiaca, hasta manifestaciones multiorgánicas y sistémicas como sepsis, choque séptico y múltiples síndromes de disfunción orgánica que requieren de oxigenación suplementaria, transfusión de plasma convaleciente, ventilación mecánica y apoyo en $\mathrm{UCI}$ (Cascella et al., 2020; Leisman et al., 2020; Rajendran et al., 2020). Hasta la fecha, los casos de COVID-19 han sido clasificados clínicamente siguiendo el orden establecido por la Comisión de la Organización Mundial de la Salud -OMS de China (2020): leve, moderado, grave y crítico, de acuerdo con la gravedad de los síntomas presentados. La tabla 1 resume el cuadro asociado a cada manifestación, según las clasificaciones presentadas por Cascella et al. (2020), Gandhi et al. (2020), Hassan et al. (2020), Hu et al. (2020) y la OMS (2020) para pacientes adultos, y por Castagnoli 
et al. (2020), Chen et al. (2020), de Souza et al. (2020), Dong et al. (2020) y la OMS (2020) para pacientes pediátricos.

En general, los datos sugieren que los niños y niñas tienen menos probabilidades de desarrollar síntomas de coronavirus que las personas adultas, con una incidencia del 1 al 5\%. Por otro lado, las investigaciones más recientes sugieren que los hombres mueren por COVID-19 a tasas más altas que las mujeres (Ludvigsson, 2020). El estudio más grande hasta el momento, desarrollado por Dong et al. (2020), incluyó el análisis de 2.143 infantes con COVID-19 documentados entre el 16 de enero y el 8 de febrero de 2020 en China; este encontró que los síntomas de la enfermedad generalmente eran menos severos en niños, niñas y adolescentes ( 1 a 17 años) en comparación con personas adultas. Sin embargo, se ha observado una prevalencia más alta de enfermedad grave en menores de un año que en casos pediátricos de mayor edad (Dong et al., 2020; Ludvigsson, 2020). Adicionalmente, evidencia reciente en el Reino Unido y los Estados Unidos de América advierte sobre un número creciente de niños y niñas (aunque aún es relativamente pequeño comparado con el total de casos de COVID-19) que presentan un síndrome hiperinflamatorio multisistémico; hasta el momento, se sugiere que podría ser una respuesta inmu- ne posinfecciosa al SARS-CoV-2, con síntomas similares a la enfermedad de Kawasaki o al síndrome de shock tóxico, que requieren hospitalización en $\mathrm{UCI}$ (Mahase, 2020; Riphagen et al., 2020).

Así pues, la evidencia disponible en la actualidad muestra que los niños y niñas de todas las edades son susceptibles a la infección por SARS-CoV-2 y que las y los neonatos parecen estar en mayor riesgo, ya que las infecciones asintomáticas son más comunes en infantes de mayor edad y en adolescentes. Aunque el curso clínico de la infección por SARS-CoV-2 en niños y niñas es más leve que en personas adultas, la mayor parte de quienes desarrollan la enfermedad COVID-19 en su manifestación grave tienen afecciones comórbidas subyacentes (Lu et al., 2020).

Las y los pacientes con cuadros graves y críticos requieren de intervención en $\mathrm{UCl}$ y $\mathrm{UCl}$-intermedio, demostrando no solo un deterioro en su salud física y mental, sino también un cambio abrupto en sus actividades básicas de la vida diaria y en su desempeño social adaptativo, lo que conlleva a desequilibrio ocupacional $^{12}$ (Gonçalo et al., 2020) y privación ocupacional ${ }^{13}$ (Provancha-Romeo et al., 2019). Dado el tratamiento médico intensivo que reciben algunos pacientes con COVID-19, incluida la ventilación pulmonar prolongada, la sedación y el

\footnotetext{
${ }^{12}$ El desequilibrio ocupacional ocurre en dos niveles: individual y social. A nivel individual, se entiende como una configuración de actividades dentro del estilo de vida de una persona que no satisface sus necesidades fisiológicas, psicológicas o sociales. A nivel social, el desequilibrio ocupacional se refiere a la distribución inequitativa de oportunidades de participación en ocupaciones significativas, beneficiosas o valoradas (Durocher, 2017).

${ }^{13}$ Se entiende como privación ocupacional a la exclusión de la participación en ocupaciones significativas, que promueven la salud o que son necesarias para la persona, debido a fuerzas más allá del control del individuo (Whiteford, 2000). Las barreras que contribuyen a la privación ocupacional incluyen: inmovilidad, privación y sobrecarga sensorial, ventilación mecánica, debilidad adquirida en la UCl, agitación, estrés y delirio (Provancha-Romeo et al., 2019).
} 
Tabla 1. Clasificación y manifestaciones clínicas de la enfermedad COVID-19.

\begin{tabular}{|c|c|c|}
\hline COVID-19 & Población adulta y adulta mayor & Población infantil \\
\hline Asintomático & \multicolumn{2}{|c|}{$\begin{array}{l}\text { Sin signos o síntomas clínicos. Imágenes torácicas normales, mientras que la prueba de ácido } \\
\text { nucleico del SARS-CoV-2 fue positiva o el anticuerpo específico del suero fue diagnosticado } \\
\text { retrospectivamente como infección. }\end{array}$} \\
\hline Leve & $\begin{array}{l}\text { Presencia de síntomas de una infección } \\
\text { viral del tracto respiratorio superior que } \\
\text { incluyen tos seca, fiebre leve, congestión } \\
\text { nasal, dolor de garganta, dolor de cabeza, } \\
\text { dolor muscular y malestar. Ausencia de } \\
\text { síntomas graves como disnea. Sin } \\
\text { evidencias radiográficas de neumonía. } \\
\text { La mayoría de los casos (80\%) de COVID- } \\
19 \text { son de gravedad leve. Pacientes con } \\
\text { enfermedad leve pueden deteriorarse } \\
\text { rápidamente en casos graves o críticos. }\end{array}$ & $\begin{array}{l}\text { Presencia de síntomas de infección aguda del } \\
\text { tracto respiratorio superior que incluyen fiebre, } \\
\text { fatiga, mialgia, tos, dolor de garganta, secreción } \\
\text { nasal y estornudos. El examen físico muestra } \\
\text { congestión de la faringe, sin anormalidades } \\
\text { auscultatorias. Algunos casos pueden no tener } \\
\text { fiebre o solo tener síntomas digestivos como } \\
\text { náuseas, vómitos, dolor abdominal y diarrea. }\end{array}$ \\
\hline Moderado & $\begin{array}{l}\text { Presencia de síntomas respiratorios como } \\
\text { tos, dificultad para respirar y taquipnea. Sin } \\
\text { embargo, no hay signos ni síntomas de } \\
\text { enfermedad grave. Puede haber evidencia } \\
\text { radiográfica de neumonía leve. }\end{array}$ & $\begin{array}{l}\text { Presencia de neumonía. Fiebre y tos frecuentes, } \\
\text { en su mayoría tos seca, seguida de tos } \\
\text { productiva. Algunos casos pueden presentar } \\
\text { jadeo, pero no hay hipoxemia obvia como falta } \\
\text { de aliento; los pulmones pueden escucharse con } \\
\text { esputo o ronquidos secos o húmedos. Algunos } \\
\text { casos pueden no tener signosy síntomas clínicos, } \\
\text { pero la tomografía computarizada de tórax } \\
\text { muestra lesiones pulmonares, que son } \\
\text { subclínicas. }\end{array}$ \\
\hline Grave & $\begin{array}{l}\text { Presencia de neumonía grave, síndrome de } \\
\text { dificultad respiratoria aguda, sepsis o shock } \\
\text { séptico (15\% de los casos). } \\
\text { El diagnóstico es clínico y las } \\
\text { complicaciones pueden excluirse con la } \\
\text { ayuda de estudios radiográficos; } \\
\text { requerimiento de oxígeno suplementario. } \\
\text { Presencia de disnea, frecuencia respiratoria } \\
\geq 30 / \text { min, saturación de oxígeno en la } \\
\text { sangre (SpO2) } \leq 93 \% \text {, relación PaO2/FiO2 } \\
\text { o P/F [la relación entre la presión sanguínea } \\
\text { del oxígeno (presión parcial de oxígeno, } \\
\text { PaO2) y el porcentaje de oxígeno } \\
\text { suministrado (fracción de oxígeno } \\
\text { inspirado, FiO2)] <300, o infiltrados } \\
\text { pulmonares > 50\% en } 24 \text { a } 48 \text { horas. } \\
\text { Incluso en las formas graves de la } \\
\text { enfermedad, la fiebre puede estar ausente } \\
\text { o ser moderada. }\end{array}$ & $\begin{array}{l}\text { Los síntomas respiratorios tempranos, como } \\
\text { fiebre y tos, pueden ir acompañados de síntomas } \\
\text { gastrointestinales como diarrea. La enfermedad } \\
\text { generalmente progresa alrededor de una semana } \\
\text { y se produce disnea, con cianosis central. } \\
\text { Presencia de taquipnea; respiración dificultosa, } \\
\text { cianosis y apnea intermitente; letargo y } \\
\text { convulsión; dificultad para alimentar y signos de } \\
\text { deshidratación. } \\
\text { Cumple cualquiera de los siguientes criterios: (1) } \\
\text { aumento de la frecuencia respiratoria: } \geq 30 \\
\text { veces/min; (2) saturación de oxígeno } \leq 93 \% \text { en } \\
\text { estado de reposo, y (3) presión parcial de oxígeno } \\
\text { arterial }(\mathrm{PaO} 2) / \text { concentración de oxígeno (FiO2) } \\
\leq 300 \mathrm{mmHg}(1 \mathrm{mmHg}=0.133 \mathrm{kPa} \text { ). }\end{array}$ \\
\hline Crítico & $\begin{array}{l}\text { Presencia de insuficiencia respiratoria, } \\
\text { lesión cardiaca, shock séptico o disfunción } \\
\text { o falla orgánica múltiple ( } 5 \% \text { de los casos). } \\
\text { Requerimiento de ventilación mecánica y } \\
\text { soporte vital. Datos de los Centros para el } \\
\text { Control y la Prevención de Enfermedades } \\
\text { de China sugieren una tasa de mortalidad } \\
\text { en pacientes críticos del } 49 \% \text {. Pacientes } \\
\text { con comorbilidades preexistentes, como } \\
\text { diabetes, enfermedad respiratoria, } \\
\text { enfermedad cardiovascular, hipertensión y } \\
\text { complicaciones oncológicas, tienen tasas } \\
\text { de letalidad más altas respecto a aquellos } \\
\text { que no las presentan. }\end{array}$ & $\begin{array}{l}\text { Los niños y niñas pueden progresar rápidamente } \\
\text { al síndrome de dificultad respiratoria aguda } \\
\text { (SDRA) o insuficiencia respiratoria, y también } \\
\text { pueden tener shock, encefalopatía, lesión } \\
\text { miocárdica o insuficiencia cardíaca, disfunción } \\
\text { de la coagulación y lesión renal aguda, incluida } \\
\text { la disfunción de múltiples órganos. Puede ser } \\
\text { mortal. Cumple cualquiera de los siguientes } \\
\text { criterios: (1) insuficiencia respiratoria que } \\
\text { requiere ventilación mecánica; (2) shock séptico, } \\
\text { y (3) acompañado de otra falla orgánica que } \\
\text { necesita monitoreo y tratamiento en la UCI. }\end{array}$ \\
\hline
\end{tabular}

Fuente: elaboración propia a partir de los autores antes citados. 
uso de agentes de bloqueo neuromuscular, quienes ingresan a la $\mathrm{UCl}$ pueden tener un alto riesgo de desarrollar DAUCl; esto puede empeorar su condición de salud y aumentar la mortalidad (Herridge et al., 2011). Hasta la fecha, se han reportado síntomas como disfagia, debilidad muscular, miopatía y neuropatía críticas, movilidad reducida de las articulaciones, dolor de cuello y hombro (debido a pronación prolongada), dificultad en la verticalización, equilibrio y marcha alterados, limitaciones en las actividades básicas de la vida diaria, dificultad para asumir un estado de alerta, estado confusional duradero y problemas psicológicos (Kiekens et al., 2020).

Así pues, el objetivo principal de la rehabilitación temprana en la $\mathrm{UCl}$ y la $\mathrm{UCl}$-intermedio debe enfocarse en minimizar los efectos adversos de la enfermedad COVID-19 en el sistema respiratorio, cardiovascular y musculoesquelético, y en el desempeño neuropsicológico; restaurar, en primera instancia, la función respiratoria para la participación en actividades básicas de la vida diaria; prevenir el desacondicionamiento físico, la privación sensorial y el deterioro cognitivo, mental y social; mejorar la independencia funcional, evitando la necesidad de ventilación mecánica u hospitalizaciones posteriores, y mejorar la calidad de vida de la persona hospitalizada (Acosta et al., 2020; Yu et al., 2020).

\section{Rol del terapeuta ocupacional en el tratamiento de pacientes con COVID-19 en fase aguda}

En esta fase se encuentran las y los pacientes confirmados o con sospecha viral de COVID-19, ventilados mecáni- camente, sedados, relajados o con riesgo de falla ventilatoria, cuyo estado de conciencia se ha conservado, pero tienen dependencia funcional. Se ha observado que la duración de la estadía en la $\mathrm{UCl}$ es más larga de lo habitual, con un promedio de tres semanas. La fase de transición de ventilación mecánica a uso de oxígeno suplementario y la transferencia a un servicio de rehabilitación deben ser graduales, con un monitoreo preciso, ya que estas personas permanecen inestables por varios días después de la extubación (Kiekens et al., 2020); en esta fase es posible que no toleren sesiones de más de 5 minutos, debido a su rápida desaturación y a la fatiga extrema. Así pues, es esencial que la o el terapeuta ocupacional evalúe los riesgos y los beneficios, y esté al tanto de las funciones de los sistemas respiratorio, nervioso, cardiovascular, hematológico e inmune (Gonçalo et al., 2020).

\section{Manejo terapéutico}

Aunque el manejo varía de acuerdo con las particularidades de cada paciente, las acciones aquí planteadas indican los aspectos generales para tener en cuenta en la intervención de Terapia Ocupacional, a partir de los resultados de la evaluación clínica integral.

\section{Acciones de intervención directa:}

- Posicionar en cama, facilitando una postura vertical. Proveer ayudas de posicionamiento en cama para facilitar la eliminación efectiva de las secreciones y la expansión pulmonar, con monitoreo permanente de los signos vitales. 
- Tomar medidas antiedema y de cuidado de la piel para la prevención de úlceras por presión.

- Incrementar la tolerancia a la actividad a través de la participación gradual, en sincronía con los signos y síntomas de disnea (no se debe permitir una saturación de $\mathrm{O}_{2}$ percutánea $<90 \%$ o cambio desde línea base $>4 \%$ ).

- Facilitar la movilización temprana: movilización pasiva o activo-asistida, generalmente en cama, prestando especial atención a la facilitación de la expectoración durante las prácticas terapéuticas o las ABVD (ej., alimentación y acicalado).

- Facilitar la estimulación multisensorial para intervenir en los diferentes grados de alteración de la conciencia (en el proceso del despertar, la disminución de la sedación) y previniendo la privación sensorial.

- Manejar medidas no farmacológicas para la prevención y el manejo del delirio.
- Implementar herramientas de comunicación para pacientes conscientes con intubación orotraqueal.

\section{Acciones de intervención indirecta:}

- Revisar registros clínicos.

- Educar al equipo sobre seguridad del paciente (posicionamiento en prono $^{14}$, medidas de confort, acojinamiento para el manejo y la disminución de edema, evitar posiciones antálgicas, prevenir zonas de presión, entre otras).

- Realizar estimulación multisensorial de acuerdo con las posibilidades del ambiente (visual, auditiva, táctil, propioceptiva, etc.), con el objetivo de obtener diferentes respuestas o reacciones del paciente, promover la conciencia interoceptiva ${ }^{15}$ y la interacción con el medio externo. De esta manera, fomentar formas de comunicación con la o el paciente y aumentar su nivel de comprensión y de control sobre su cuerpo y el ambiente que le rodea.

\footnotetext{
${ }^{14}$ El posicionamiento en prono, también conocido como ventilación en prono, es una estrategia recomendada en pacientes adultos con $\mathrm{PaO} 2 / \mathrm{FiO} 2<150$, para mejorar la mecánica pulmonar y la oxigenación. Esta estrategia ha sido utilizada con anterioridad a la pandemia para facilitar la oxigenación en pacientes con síndrome respiratorio grave agudo (Scholten et al., 2017). Generalmente se mantiene a la persona en prono por 12 a 16 horas. La ventilación en prono puede cesar una vez que la PaO2/FiO2 es $>150$ por más de 4 horas en posición supina. Sin embargo, en entornos con recursos limitados, como disponibilidad de personal o de equipos de protección personal, puede aumentar innecesariamente el riesgo de infección para las y los trabajadores de la salud (Sundaram et al., 2020). Se ha evidenciado que el mantenimiento de la posición en prono por tiempos prolongados está asociado a riesgos de compresión neural y vascular; en consecuencia, es necesario prevenir y tratar oportunamente las lesiones de nervio periférico que pueden resultar del mismo, interfiriendo en el funcionamiento de los miembros superiores (Bouyer-Ferullo, 2013; Spruce y Van Wicklin, 2014).

${ }^{15}$ La conciencia interoceptiva se refiere al proceso sensorial de recibir, acceder y evaluar las señales corporales internas, tales como el latido cardíaco, la respiración, la saciedad y la actividad del sistema nervioso autónomo. Esta conciencia influye en la autorregulación de las emociones y en la salud mental (Critchley et al., 2004; Gafinkel et al., 2015; Price y Hooven, 2018).
} 
- Favorecer habilidades cognitivas básicas de memoria, atención, comprensión y orientación hacia el ambiente.

- Identificar y aproximarse al núcleo familiar (de manera no presencial) para realizar un perfil ocupacional que contenga un repertorio ocupacional significativo y elementos biográficos que potencien el reconocimiento integral de la persona y la construcción de un vínculo terapéutico durante el proceso de atención. Se tienen en cuenta la historia y las experiencias ocupacionales, los patrones de la vida diaria, los intereses, los valores y las necesidades, entre otros aspectos.

- Empoderar a la familia e involucrarla en el proceso terapéutico a través de herramientas y estrategias que promuevan la estimulación multisensorial, como el uso de calendarios, cartas, fotos, entre otro material que pueda luego descartarse. Estos podrán ser ingresados al cuarto una vez la o el paciente salga de la sedación, para brindar un entorno físico humanizado y personalizado.

- Considerar estrategias para el manejo de la ansiedad en las y los familiares.

- Entrenar al equipo de tratamiento, así como a cuidadores y familiares en el uso de alternativas para facilitar la comunicación alternativa, de acuerdo con las necesidades y las posibilidades del ambiente (ej., a través de videochat, grabaciones de voz, cartas).

\section{Rol del terapeuta ocupacional en el tratamiento de pacientes con COVID-19 en fase subaguda}

En esta fase se encuentran las y los pacientes con COVID-19 que han superado el síndrome respiratorio agudo y quienes se encuentran hemodinámicamente estables y concientes, requiriendo manejo clínico en la UCl-intermedio.

\section{Manejo terapéutico}

Aunque el manejo varía de acuerdo con las particularidades de cada paciente, las acciones aquí planteadas indican los aspectos generales para tener en cuenta en la intervención de Terapia Ocupacional, a partir de los resultados de la evaluación clínica integral y del progreso desde la $\mathrm{UCI}$.

En esta fase es importante observar el desempeño de la persona durante las actividades correspondientes a las ocupaciones que desea realizar, identificando la eficacia de la intervención en el mejoramiento de las destrezas y patrones de ejecución; identificar y medir de manera más específica los contextos o entornos, las demandas de la actividad y las características de la persona; interpretar los datos de evaluación para identificar los apoyos necesarios y los obstáculos para su desempeño, y considerar estrategias para la recuperación, teniendo en cuenta el nivel de intervención correspondiente (ej., prevención, promoción, rehabilitación).

\section{Acciones de intervención directa}

- Comunicar con el equipo de trabajo, cuidadores y familiares, los niveles de funcionalidad dentro de 
las actividades básicas de la vida diaria y otras actividades significativas para la o el paciente, con miras a establecer planes de intervención seguros y eficaces.

- Evaluar y manejar las alteraciones en el área motora (arcos de movimiento, fuerza muscular, patrones funcionales e integrales, coordinación, equilibrio y ajuste postural, marcha durante actividades básicas de la vida diaria), el procesamiento cognitivo (atención, comprensión, orientación, memoria, esquema y conciencia corporal, análisis y solución de problemas, secuencias, velocidad en el procesamiento de la información, capacidad visoespacial y la función ejecutiva) y la interacción con el ambiente.

- Evaluar la funcionalidad del paciente, prestando especial atención a su relación con el dolor (ej., a través de la escala visual analógica del dolor -EAV), y monitorear su evolución durante la participación en actividades, así como los signos y síntomas de disnea asociados (ej., a través de la escala de disnea de Borg). En concordancia con ello, favorecer la modificación de actividades y el desprendimiento gradual de la necesidad de oxígeno suplementario, en sincronía con las acciones del equipo multidisciplinario.

- Educar al paciente en la incorporación de principios de conservación de energía, estrategias de simplificación del trabajo y uso adecuado de la biomecánica del cuerpo durante las ocupaciones diarias.
- Manejar la ansiedad y prevenir el síndrome de estrés postraumático con soporte familiar y entrenamiento en técnicas de promoción de la conciencia interoceptiva (ej., haciendo uso del pulsioxímetro).

- Prevenir, detectar y monitorear el delirio.

-Facilitar la estimulación multisensorial.

- Generar un puente de comunicación con las y los familiares (visitas virtuales, diario de $\mathrm{UCl}$ donde la familia mantiene un récord de los hechos ocurridos).

- Incrementar la comprensión de la enfermedad, del proceso de recuperación y de su posible impacto en el desempeño ocupacional.

- Educar al paciente en técnicas de respiración controlada (respiración diafragmática y de labios fruncidos) y estrategias de alivio de la disnea.

- Educar al paciente en la evasión de toxinas ambientales y en minimizar el riesgo de infección (uso correcto de tapabocas, higiene, distanciamiento social).

- Educar al paciente en técnicas de relajación y manejo del estrés.

- Desarrollar habilidades para el manejo de la ansiedad relacionada con los desórdenes respiratorios y el distanciamiento físico.

\section{Acciones de Intervención indirecta}

- Organizar un horario con los periodos de atención y cuidados brin- 
dados por cada profesional, para aumentar la sensación de control de la rutina del paciente en la $\mathrm{UCl}$ - intermedio.

- Adaptar el entorno con apoyo de los elementos elaborados o proporcionados por la familia.

- Educar a familiares y cuidadores en técnicas de relajación y habilidades para el manejo de la ansiedad relacionada con el distanciamiento físico.

- Educar y entrenar a familiares y cuidadores para preparar el espacio del hogar y usarlo de acuerdo con las necesidades ocupacionales del paciente.

- Diseñar actividades terapéuticas para fortalecer habilidades intelectuales, motoras, emocionales y funcionales.

- Diseñar actividades lúdicas y de esparcimiento con el propósito de canalizar el estado emocional que se puede presentar por el proceso de enfermedad, el aislamiento y la estancia prolongada en el hospital.

- Orientar al personal de enfermería para que, en la medida de lo posible, haga partícipe al paciente en las actividades básicas de la vida diaria.

- Promover los cambios constantes de posición, considerando principios biomecánicos tanto para la o el paciente como para el personal que lo asiste; de esta manera, prevenir mayores compromisos osteomusculares y alteraciones en la piel por el reposo prolongado, y evitar lesiones en el personal de enfermería.
- Establecer un medio de comunicación telefónica con la familia para disminuir la sensación de abandono y el aislamiento social. Si es posible, a través de plataformas, facilitar reuniones virtuales.

- Realizar actividades motoras en las diferentes posiciones, de acuerdo con las condiciones de salud del paciente; especialmente en sedestación, favoreciendo el fortalecimiento muscular, la coordinación y el equilibrio, de manera que le permitan lograr una mayor seguridad postural y participar en las actividades básicas de la vida diaria.

\section{Discusión}

El papel del terapeuta ocupacional en la atención de pacientes con COVID-19 durante las primeras etapas de la enfermedad (aguda y subaguda) es fundamental para evitar el desacondicionamiento físico y el delirio derivados del reposo prolongado, el tiempo de sedación y el aislamiento familiar y social, y para combatir la privación ocupacional. El objetivo de la Terapia Ocupacional en estas fases de hospitalización se centra en facilitar la participación en las ocupaciones, rutinas diarias y roles de vida de la persona, dentro de su rango de tolerancia. Sin embargo, es necesario que las y los terapeutas ocupacionales que trabajan en la atención hospitalaria en las fases aguda y subaguda, además de contar con su título profesional, tengan entrenamiento y experiencia en $\mathrm{UCl}$ y UCl-intermedio, así como conocimiento y manejo de los equipos propios de estas unidades. 
Aunque hasta ahora sean excepcionales los casos de niñas, niños y neonatos contagiados por el COVID-19, es necesario tener en cuenta que esta condición podría agudizar las comorbilidades ya presentes en estas poblaciones, por lo que no se debería excluir la intervención temprana brindada por Terapia Ocupacional en las $\mathrm{UCI}$ neonatal y $\mathrm{UCI}$ pediátrica, siempre y cuando se cuente con las medidas de bioseguridad reglamentadas por el Ministerio de Salud y Protección Social. Así mismo, se debe tener en cuenta que, hasta la fecha, las personas adultas y adultas mayores más vulnerables a desarrollar la enfermedad COVID-19 grave y crítica son aquellas con comorbilidades, entre ellas, quienes tienen discapacidades. Como lo señala la Oficina del Alto Comisionado de las Naciones Unidas para los Derechos Humanos (2020), a pesar de estar especialmente expuestas al COVID-19, las personas con discapacidades enfrentan incluso mayores desigualdades en la atención en salud durante la pandemia, debido a las barreras de acceso a la información y a los entornos sanitarios, así como a la existencia de pautas y protocolos que pueden aumentar la discriminación hacia ellas. En este sentido, es necesario que la atención hospitalaria en las fases aguda y subaguda esté enmarcada bajo los principios de igualdad y no discriminación, y garantice el acceso equitativo, seguro y oportuno a la rehabilitación temprana.

Por otro lado, el personal que se considere de alto riesgo no debe ser forzado, en ninguna circunstancia o bajo ninguna medida, a entrar en el área de aislamiento de pacientes con COVID-19. En la asignación y planeación de personal, debe tenerse en cuenta a quienes, en caso de resultar contagiados, presentan un mayor riesgo de desarrollar enfermedades más graves; en consecuencia, se debe evitar su contacto con pacientes con COVID-19 (Thomas et al., 2020). Se incluyen aquellos miembros del equipo de salud que:

- Estén embarazadas.

- Tengan enfermedades respiratorias crónicas relevantes.

-Estén inmunodeprimidos.

-Sean mayores de 60 años.

- Tengan enfermedades crónicas graves, como condiciones cardiovasculares o pulmonares, o diabetes.

-Tengan deficiencias inmunitarias, como neutropenia, neoplasia maligna diseminada y afecciones o tratamientos que produzcan una inmunodeficiencia.

Para terapeutas ocupacionales que trabajan con pacientes con COVID-19 es preciso:

- Utilizar correctamente todos los elementos de protección, siguiendo los respectivos protocolos institucionales.

- Realizar la higiene de manos contemplando los cinco momentos exigidos por la OMS para la atención de pacientes (OMS, 2010).

- Realizar las debidas desinfecciones, previa y posterior a la intervención, de los elementos terapéuticos, teniendo en cuenta los protocolos institucionales. 
- Monitorear los signos vitales antes, durante y después de la actividad, ya que reflejan la función cardiopulmonar del paciente.

- Observar atentamente los signos de cianosis, empalidecimiento, hipocratismo digital de manos y pies, uso de músculos accesorios o patrones de respiración anormales.

- Modificar las actividades si la saturación de oxígeno cae por debajo del 90\% (u otros parámetros establecidos por el cuerpo médico de la unidad).

- Asegurar que las y los pacientes mantengan una buena higiene oral, esto reduce la colonización bacteriana que puede llevar a desarrollar neumonía adquirida en el ambiente hospitalario. La higiene oral incluye el cepillado de dientes y lengua, y el uso de enjuague antiséptico bucal. A algunas personas se les puede haber prescrito un enjuague bucal especial (por ejemplo, cloredexina).

- Respetar todas las precauciones de aspiración y deglución consignadas en el registro médico del paciente o del cuarto.

- Trabajar en equipo con el grupo multidisciplinario, promoviendo el bienestar mental, emocional y físico del paciente, y conservando al máximo la participación y la independencia funcional.

\section{Conclusión}

Los presentes lineamientos constituyen un recurso técnico y educativo relevante para la discusión y el desarrollo de la Terapia Ocupacional hospitalaria en Colombia. Estos lineamientos están sujetos a las revisiones y los avances en el conocimiento científico respecto a la enfermedad COVID-19, así como a los cambios que ello genere en las prácticas en salud y rehabilitación temprana, que sin duda seguirán transformando el panorama de salud a nivel local y global.

\section{Referencias}

Acosta, M., Ariza, M., Arribas, A.M., Blásquez, V., Fernández, J., Gómez, C., Herrera, D., Incio, M.J., Lastres, A., Martín, M., Martínez, N., Martínez, S., Moreno, M., Muñoz, V., Pérez, J. y Sancho, C. (2020). Guía clínica de intervención de terapia ocupacional en pacientes con COVID-19. Colegio Profesional de Terapeutas Ocupacionales de la Comunidad de Madrid [COPTOCAM]. https://coptocam.org/wp-content/ uploads $/ 2020 / 05 / \mathrm{Gu} \% \mathrm{C} 3 \%$ ADa-cl\%C3\%ADnica-de-TO-covid-19-.pdf

Algeo, N. y Aitken, L. M. (2019). The evolving role of occupational therapists in adult critical care in England. Irish Journal of Occupational Therapy, 42(7), 74-94. http:// doi.org/10.1108/IJOT-04-2019-0005

Álvarez, E. A., Garrido, M. A., Tobar, E. A., Prieto, S. A., Vergara, S. O., Briceño, C. D. y González, F. J. (2017). Occupational therapy for delirium management in elderly patients without mechanical ventilation in an intensive care unit: A pilot randomized clinical trial. Journal of critical care, 37, 85-90. https:// doi.org/10.1016/j.jcrc.2016.09.002 
Anekwe, D. E., Biswas, S., Bussières, A. y Spahija, J. (2019). Early rehabilitation reduces the likelihood of developing intensive care unit-acquired weakness: A systematic review and meta-analysis. Physiotherapy, 107, 1-10. https://doi.org/10.1016/j.physio.2019.12.004

Benavides, A., Castillo, G., Landauro, M. y Vásquez, G. (2006). Factores que prolongan la estancia hospitalaria en el Hospital Nacional PNP Luis N. Sáenz. Revista de Medicina Humana-Universidad Ricardo Palma, 6(2), 3-12.

Betters, K. A., Hebbar, K. B., Farthing, D., Griego, B., Easley, T., Turman, H., Perrino, L., Sparacino, S. y de Almeida, M. L. (2017). Development and implementation of an early mobility program for mechanically ventilated pediatric patients. Journal of critical care, 41, 303-308. https://doi.org/10.1016/j.jcrc.2017.08.004

Berrios, S. G., Álvarez, J. C., Gimpel, V. E., Bolaño, G. F. y Toro, K. S. (2019). Rol del terapeuta ocupacional en el tratamiento de niños con delirium hospitalario infantil en unidades hospitalarias de Chile. Revista Chilena de Terapia Ocupacional, 19(1), 69-82. https://ultimadecada.uchile. cl/index.php/RTO/article/view/53685

Bouyer-Ferullo, S. (2013). Preventing perioperative peripheral nerve injuries. AORN journal, 97(1), 110-124. https:// doi.org/10.1016/j.aorn.2012.10.013

Borges Nery, P., Snider, L., Camelo, J. S., Boychuck, Z., Khodary, F., Goldschleger, J. y Majnemer, A. (2019). The role of rehabilitation specialists in Canadian nicus: a 21st century perspective. Physical \& occupational therapy in pediatrics, 39(1), 33-47. https://doi. org/10.1080/01942638.2018.1490846
Cascella, M., Rajnik, M., Cuomo, A., Dulebohn, S. C. y Di Napoli, R. (2020). Features, evaluation and treatment coronavirus (COVID-19). En Statpearls [internet]. StatPearls Publishing. https://www.ncbi. nlm.nih.gov/books/NBK554776/

Castagnoli, R., Votto, M., Licari, A., Brambilla, I., Bruno, R., Perlini, S., Rovida, F., Baldanti, F. y Marseglia, G. L. (2020). Severe Acute Respiratory Syndrome Coronavirus 2 (SARS-CoV-2) infection in children and adolescents: A systematic review. JAMA pediatrics. https://doi.org/10.1001/jamapediatrics.2020.1467

Colegio Colombiano de Terapia Ocupacional [CCTO] (2020, 29 de abril). Pronunciamiento sobre el rol de la Terapia Ocupacional en la atención hospitalaria durante la pandemia por COVID-19. https://6e05f2cf-f3fc-4799-b443-264a49ac10ee.filesusr.com/ugd/7ce81a c46fe1c$\underline{221294 f c 68 f 41 b 90 f a 7 d 808 b 5 . p d f}$

Chen, A., Huang, J., Liao, Y., Liu, Z., Chen, D., Yang, C., Yang, R. y Wei, X. (2020). Differences in Clinical and Imaging Presentation of Pediatric Patients with COVID-19 in Comparison with Adults. Radiology: Cardiothoracic Imaging, 2(2), e200117. https://doi.org/10.1148/ryct.2020200117

Coelho Filho, J. M. (2000). Modelos de serviços hospitalares para casos agudos em idosos. Revista de Saúde Pública, 34(6), 666-671. https://doi.org/10.1590/S003489102000000600017

Corcoran, J.R., Herbsman, J. M., Bushnik, T., Van Lew, S., Stolfi, A., Parkin, K., McKenzie, A., Hall, G., Joseph, W., Whiteson, J. y Flanagan, S.R. (2017). Early rehabilitation in the medical and surgical intensive care units for patients with and without mechanical ventilation: an interprofes- 
sional performance improvement project. $P M \& R, 9(2), 113-119$. https://doi. org/10.1016/j.pmrj.2016.06.015

Costigan, F. A., Duffett, M., Harris, J. E., Baptiste, S. y Kho, M. E. (2019). Occupational therapy in the ICU: A scoping review of 221 documents. Critical care medicine, 47(12), e1014-e1021. https://doi. org/10.1097/CCM.0000000000003999

Critchley, H. D., Wiens, S., Rotshtein, P., Öhman, A. y Dolan, R. J. (2004). Neural systems supporting interoceptive awareness. Nature neuroscience, 7(2), 189-195. https://doi.org/10.1038/nn1176

Cuevas-Lara, C., Izquierdo, M., Gutiérrez-Valencia, M., Marín-Epelde, I., Zambom-Ferrasi, F., Contreras-Escámez, B. y Martínez-Velilla, N. (2019). Effectiveness of occupational therapy interventions in acute geriatric wards: a systematic review. Maturitas, 127, 43-50. https://doi. org/10.1016/j.maturitas.2019.06.005

Cuello-Garcia, C. A., Mai, S. H. C., Simpson, R., Al-Harbi, S. y Choong, K. (2018). Early mobilization in critically ill children: a systematic review. The Journal of pediatrics, 203, 25-33. https://doi.org/10.1016/j.jpeds.2018.07.037

Cui, L. R., LaPorte, M., Civitello, M., Stanger, M., Orringer, M., Casey III, F., Kuch, B., Beers, S., Valenta, C., Kochanek, P., Houtrow, A. y Fink, E. (2017). Physical and occupational therapy utilization in a pediatric intensive care unit. Journal of critical care, 40, 15-20. https://doi.org/10.1016/j.jcrc.2017.03.003

Dong, Y., Mo, X., Hu, Y., Qi, X., Jiang, F., Jiang, Z. y Tong, S. (2020). Epidemiological characteristics of 2143 pediatric patients with 2019 coronavirus disease in
China. Pediatrics [pre-print]. https://doi. org/10.1542/peds.2020-0702

Durocher, E. (2017). Occupational justice: A fine balance for occupational therapists. In D. Sakellariou y N. Pollard (Eds.). Occupational Therapies Without Borders. Integrating Justice with Practice (pp. 9-18). Elsevier.

Ferrante, L. E., Murphy, T. E., Leo-Summers, L. S., Gahbauer, E. A., Pisani, M. A. y Gill, T. M. (2019). The combined effects of frailty and cognitive impairment on post-ICU disability among older ICU survivors. American journal of respiratory and critical care medicine, 200(1), 107-110. https://doi.org/10.1164/rccm.201806-1144LE

Fink, E., Beers, S., Houtrow, A.., Richichi, R., Burns, C., Doughty, L., Ortiz, R., Madurski, C., Valenta, C., Chrisman, M., Golightly, L., Kiger, M., Patrick, C., Treble, A., PoIlon, D., Smith, C., Kochanek, P. (2019). Early protocolized versus usual care rehabilitation for pediatric neurocritical care patients: a randomized controlled trial. Pediatric Critical Care Medicine, 20(6), 540-550. https://doi.org/10.1097/ PCC.0000000000001881

Gandhi, R. T., Lynch, J. B. y del Rio, C. (2020). Mild or moderate COVID-19. New England Journal of Medicine. https:// doi.org/10.1056/NEJMcp2009249

Garfinkel, S. N., Seth, A. K., Barrett, A. B., Suzuki, K. y Critchley, H. D. (2015). Knowing your own heart: distinguishing interoceptive accuracy from interoceptive awareness. Biological psychology, 104, 65-74. https://doi.org/10.1016/j. biopsycho.2014.11.004 
Gonçalo, T., Santos Nascimento, J., Barbieri Bombarda, T., Vieria Espalenza, G., Almeida Rodrigues, E.A., Ferreira, A.P. y Rodrigues dos Santos, Z. (2020). Terapia Ocupacional em cuidados paliativos na COVID-19. Comitê de Terapia Ocupacional da Academia Nacional de Cuidados Paliativos, ANCP.

Hanna, E.S., Zhao, S., Shannon, C.N. y Betters, K.A. (2020). Changes in provider perceptions regarding early mobility in the PICU. Pediatric Critical Care Medicine| Society of Critical Care Medicine, 21(1), e30-e38. https://doi.org/10.1097/ PCC.0000000000002177

Hassan, S.A., Sheikh, F.N., Jamal, S., Ezeh, J.K. y Akhtar, A. (2020). Coronavirus (COVID-19): a review of clinical features, diagnosis, and treatment. Cureus, 12(3). https://doi.org/10.7759/cureus.7355

Herrup, E. A., Wieczorek, B. y Kudchadkar, S. R. (2017). Characteristics of postintensive care syndrome in survivors of pediatric critical illness: A systematic review. World journal of critical care medicine, 6(2), 124. https:// doi.org/10.5492/wjccm.v6.i2.124

Hollinger, A., Gayat, E., Féliot, E., Paugam-Burtz, C., Fournier, M.C., Duranteau, J., Lefrant, J.Y., Leone, M., Jaber, S., Mebazaa, A. y Arrigo, M. (2019). Gender and survival of critically ill patients: results from the FROG-ICU study. Annals of intensive care, 9(1), 43. https://doi. org/10.1186/s13613-019-0514-y

Hopkins, R. O., Choong, K., Zebuhr, C. A. y Kudchadkar, S. R. (2015). Transforming PICU culture to facilitate early rehabilitation. Journal of Pediatric Intensive Care, 4(04), 204-211. https://doi.org/10.1055/s-0035-1563547
Hu, Z., Song, C., Xu, C., Jin, G., Chen, Y., $\mathrm{Xu}, \mathrm{X} ., \mathrm{Ma}, \mathrm{H} .$, Chen, Y., Lin, Y., Zheng, Y., Wang, J., Hu, Z., Yi, Y. y Shen, H. (2020). Clinical characteristics of 24 asymptomatic infections with COVID-19 screened among close contacts in Nanjing, China. Science China Life Sciences, 1-6. https://doi.org/10.1007/ s11427-020-1661-4

Kaur, S., Silver, G., Samuels, S., Rosen, A., Weiss, M., Mauer, E., Gerber, L., Greenwald, B. y Traube, C. (2020). Delirium and developmental disability: Improving specificity of a pediatric delirium screen. Pediatric Critical Care Medicine, 21(5), 409-414. https://doi.org/10.1097/ PCC.0000000000002248

Kiekens, C., Boldrini, P., Andreoli, A., Avesani, R., Gamna, F., Grandi, M., Lombardi, F., Lusuardi, M., Molteni, F., Perboni, A. y Negrini, S. (2020). Rehabilitation and respiratory management in the acute and early post-acute phase." Instant paper from the field" on rehabilitation answers to the Covid-19 emergency. European Journal of Physical and Rehabilitation Medicine, 06305-4. https://doi. org/10.23736/S1973-9087.20.06305-4

Kho, M.E., Brooks, D., Namasivayam-MacDonald, A., Sangrar, R. y Vrkljan, B. (2020) Rehabilitation for patients with COVID-19. Guidance for occupational therapists, physical therapists, speech-language pathologists and assistants. School of Rehabilitation Science, McMaster University. https://srs-mcmaster.ca/covid-19/

Legg, L., Drummond, A. y Langhorne, P. (2006). Occupational therapy for patients with problems in activities of daily living after stroke. Cochrane Database of Systematic Reviews, (4). https://doi. org/10.1002/14651858.CD003585.pub2 
Leisman, D. E., Deutschman, C. S. y Legrand, M. (2020). Facing COVID-19 in the ICU: vascular dysfunction, thrombosis, and dysregulated inflammation. Intensive Care Medicine. https://doi.org/10.1007/s00134-020-06059-6

Lima, E.M.F.D.A., Okuma, D.G. y Pastore, M.D.N. (2013). Atividade, ação, fazer e ocupação: a discussão dos termos na Terapia Ocupacional brasileira. Cadernos daTerapia Ocupacional UFSCar, 21(2), 243254. https://doi.org/10.4322/cto.2013.026

Lu, X., Xiang, Y., Du, H. y Wing-Kin Wong, G. (2020). SARS-CoV-2 infection in children-understanding the immune responses and controlling the pandemic. Pediatric Allergy and Immunology, 0, 1-5. https://doi.org/10.1111/pai.13267

Ludvigsson, J. F. (2020). Systematic review of COVID-19 in children show milder cases and a better prognosis than adults. Acta Paediatrica, 109, 1088-1095. https://doi. org/10.1111/apa.15270

Marrugo-Arnedo, C. A., Arrieta-Arrieta, A., Herrera-Malambo, D., Díaz-Vargas, L. C., Pérez-Yepes, C., Dueñas-Castell, C., Flórez-Tanus, A., Gómez, F., Alvear-Pájaro, R. y Alvis-Guzmán, N. (2019). Determinantes de estancia prolongada de neonatos en una unidad de cuidados intensivos. Revista Ciencias de la Salud, 17(2), 259-275. https://doi.org/10.12804/revistas.urosario. edu.co/revsalud/a.7928

Martell, M., Burgueño, M., Arbón, G., Weinberger, M., Balbi, C., Munyo, A., Martinotti, L., Murillo, S., Keshishian, R., Pomi, A. y Alonso, R. (2007). Asociación entre morbilidad neonatal y desarrollo en pretérminos a la edad escolar. Archivos de Pediatría del Uruguay, 78(2), 99-109. https://www.sup.org.uy/archivos-de-pediatria/adp78-2/pdf/adp78-2 3.pdf
Moreno-Chaparro, J., Cubillos-Mesa, C. y Duarte-Torres, S. C. (2019). Rol de terapia ocupacional en la unidad de cuidado intensivo en Colombia. Revista Ciencias de la Salud, 17(1), 70-84. https://doi. org/10.12804/revistas.urosario.edu.co/ revsalud/a.7614

Needham, D., Davidson, J., Cohen, H., Hopkins, R., Weinert, C., Wunsch, H., Zawistowski, C., Dougherty, A., Berney, S., Bienvenu, O., Brady, S., Brodsky, M., Denehy, L., Elliott, D., Flatley, C., Harabin, A., Jones, C., Louis, D. Meltzer, W.... y Brady, S. L. (2012). Improving long-term outcomes after discharge from intensive care unit: report from a stakeholders' conference. Critical care medicine, 40(2), 502-509. https://doi.org/10.1097/ CCM.0b013e318232da75

Oficina del Alto Comisionado en Derechos Humanos de la ONU (2020, abril). COVID-19 and the rights of persons with disabilities: guidance. https://www. ohchr.org/Documents/Issues/Disability/ COVID-19 and The Rights of Persons with Disabilities.pdf

Organización Mundial de la Salud [OMS] (2010, octubre). Sus 5 momentos para la higiene de las manos. Seguridad del Paciente. https://www.who.int/gpsc/ information centre/gpsc 5 momentos poster_es.pdf?ua $=1$

Organización Mundial de la Salud [OMS] (2020, febrero). Report of the WHO-China Joint Mission on Coronavirus Disease 2019 (COVID-19). https://www.who. int/docs/default-source/coronaviruse/ who-china-joint-mission-on-covid-19-final-report.pdf 
Pickens, N. D. y Pizur-Barnekow, K. (2009). Co-occupation: Extending the dialogue. Journal of Occupational Science, 16(3), 151-156. https://doi.org/10.1080/144275 $\underline{91.2009 .9686656}$

Pierce, D. (2001). Untangling occupation and activity. American Journal of Occupational Therapy, 55(2), 138-146. https:// doi.org/10.5014/ajot.55.2.138

Popovich, K. (2011). Chapter 2: The intensive care unit. En H. Smith-Gabai (Ed.) Occupational Therapy in Acute Care (pp. 4174). AOTA Press.

Pozzi, C., Tatzer, V. C., Álvarez, E. A., Lanzoni, A. y Graff, M. J. (2020). The applicability and feasibility of occupational therapy in delirium care. European Geriatric Medicine, 1-8. https://doi.org/10.1007/ s41999-020-00308-z

Price, C.J., y Hooven, C. (2018). Interoceptive awareness skills for emotion regulation: Theory and approach of mindful awareness in body-oriented therapy (MABT). Frontiers in psychology, 9, 798. https:// doi.org/10.3389/fpsyg.2018.00798

Provancha-Romeo, A. F., Hoffman, A. L., Malcolm, M. P., Coatsworth, J. D., Laxton, L. R., Freeman, K. M. y Schmid, A. A. (2019). Mind-body interventions utilized by an occupational therapist in a medical intensive care unit: An exploratory case study. Work (Reading, Mass.), 63(2), 191. https://doi.org/10.3233/WOR-192920

Rajendran, K., Narayanasamy, K., Rangarajan, J., Rathinam, J., Natarajan, M. y Ramachandran, A. (2020). Convalescent plasma transfusion for the treatment of COVID-19: Systematic review. Journal of Medical Virology, 1-9. https://doi. org/10.1002/jmv.25961
Reimer, C., y Slinger, P. (2019). Intraoperative patient positioning and neurological injuries. En Principles and Practice of Anesthesia for Thoracic Surgery (pp. 337-342). Springer, Cham.

Rengel, K.F., Hayhurst, C.J., Pandharipande, P.P. y Hughes, C.G. (2019). Long-term cognitive and functional impairments after critical illness. Anesthesia \& Analgesia, 128(4), 772-780. https://doi. org/10.1213/ANE.0000000000004066

Riphagen, S., Gomez, X., Gonzalez-Martinez, C., Wilkinson, N. y Theocharis, P. (2020). Hyperinflammatory shock in children during COVID-19 pandemic. The Lancet. $\quad$ https://doi.org/10.1016/S01406736(20)31094-1

Ross, K., Heiny, E., Conner, S., Spener, P. y Pineda, R. (2017). Occupational therapy, physical therapy and speech-language pathology in the neonatal intensive care unit: Patterns of therapy usage in a level IV NICU. Research in developmental disabilities, 64, 108-117. https://doi.org/10.1016/j.ridd.2017.03.009

Rubio-Grillo, M. H. (2019). Desempeño profesional del terapeuta ocupacional en la unidad de cuidado intensivo neonatal. Colombia Médica, 50(1), 30-39. http:// dx.doi.org/10.25100/cm.v50i1.2600

Sabartés, O., Sánchez, D. y Cervera, A. M. (2009). Unidades de subagudos. Revista Española de Geriatría y Gerontología, 44, 34-38. https://doi.org/10.1016/j. regg.2009.01.008

Smith, A., Karpf, M., Jow, M., Jardon, M., Yu, T. y Hutchins, B. (2019). Mothers' experiences with infant co-occupations after NICU discharge. American Journal of Occupational Therapy, 73(4_Supplement_1), 
7311505075p1-7311505075p1. https:// doi.org/10.5014/ajot.2019.73S1-PO1014

Smith, H. A. y Williams, S. R. (2020). Pediatric delirium assessment, prevention, and management. En Delirium (pp. 73-92). Springer, Cham.

Spruce, L., y Van Wicklin, S. A. (2014). Back to basics: positioning the patient. AORN journal, 100(3), 298-305. https://doi.org/10.1016/j.aorn.2014.06.004

Sundaram, M., Ravikumar, N., Bansal, A., Nallasamy, K., Basavaraja, G. V., Lodha, R., Gupta, D., Odena, M., Ram, A., Jayashree, M. (2020). Novel coronavirus 2019 (2019-nCoV) infection: Part II-Respiratory support in the pediatric intensive care unit in resource-limited settings. Indian Pediatrics, 57, 335-342. https://doi. org/10.1007/s13312-020-1786-X

Scholten, E. L., Beitler, J. R., Prisk, G. K. y Malhotra, A. (2017). Treatment of ARDS with prone positioning. Chest, 151(1), 215-224. https://doi.org/10.1016/j.chest.2016.06.032

Schweickert, W., Pohlman, M., Pohlman, A., Nigos, C., Pawlik, A.., Esbrook, C., Spears, L., Miller, M., Franczyk, M., Deprizio, D., Schmidt, G., Bowman, A., Barr, R., McCallister, K., Hall, J. y Kress, J. (2009). Early physical and occupational therapy in mechanically ventilated, critically ill patients: a randomised controlled trial. The Lancet, 373(9678), 1874-1882. https://doi.org/10.1016/S01406736(09)60658-9

Thomas, P., Baldwin, C., Bissett, B., Boden, I., Gosselink, R., Granger, C., Hodgson, C., Jones, A., Kho, M., Moses, R., Ntoumenopoulos, G., Parry, S., Patman, S. y Lee, L. (2020). Physiotherapy management for COVID-19 in the acute hospital setting: clinical practice recommendations. Jour- nal of Physiotherapy, 66(2), 73-82. https:// doi.org/10.1016/j.jphys.2020.03.011

Treyvaud, K., Lee, K. J., Doyle, L. W. y Anderson, P. J. (2014). Very preterm birth influences parental mental health and family outcomes seven years after birth. The Journal of pediatrics, 164(3), 515-521. https://doi.org/10.1016/j.jpeds.2013.11.001

Weinreich, M., Herman, J., Dickason, S. y Mayo, H. (2017). Occupational therapy in the intensive care unit: a systematic review. Occupational therapy in health care, 31(3), 205-213. https://doi.org/10.1 080/07380577.2017.1340690

Whiteford, G. (2000). Occupational deprivation: Global challenge in the new miIlennium. British journal of occupational therapy, 63(5), 200-204. https://doi. org/10.1177/030802260006300503

Wieczorek, B., Ascenzi, J., Kim, Y., Lenker, H., Potter, C., Shata, N., Mitchel, L., Haut, C., Berkowitz, I., Pidcock, F., Hoch, J., Malamed, C., Kravitz, T. y Kudchadkar, S. (2016). PICU Up! Impact of a quality improvement intervention to promote early mobilization in critically ill children. Pediatric critical care medicine: a journal of the Society of Critical Care Medicine and the World Federation of Pediatric Intensive and Critical Care Societies, 17(12), e559. https://doi.org/10.1097/ PCC.0000000000000983

Yu, P., Wei, Q. y He, C. (2020). Early rehabilitation for critically ill patients with COVID-2019: More benefits than risks. American Journal of Physical Medicine \& Rehabilitation. https://doi. org/10.1097/PHM.0000000000001445 